\title{
Preparation Methods and Application of Silicon Oxide Films
}

\author{
Wang Guogang* \\ Beijing Guodian Futong Science and \\ Technology Development Co., Ltd \\ Beijing, China \\ e-mail:ssnabi@126.com \\ Yang Haijiao \\ Beijing Guodian Futong Science and \\ Technology Development Co., Ltd \\ Shijiazhuang Tiedao University \\ Beijing, China \\ e-mail:yanghaijiao89@163.com
}

\author{
Liang Jing \\ Shanxi Jincheng Power Supply Company \\ Jincheng, China \\ e-mail:liangjing@sx.sgcc.com.cn
}

\author{
Chen Qiang \\ Beijing Insitute of Graphic Communication \\ Beijing, China \\ e-mail:chenqiang@bigc.edu.cn
}

\begin{abstract}
Silicon oxide is widely used as a thin film to improve the surface properties of materials, because it is of anti-resistance, hardness, corrosion resistance, dielectric, optical transparency etc. Just as these excellent performances, silicon oxide thin films are used in many fields. There are many ways to get silicon oxide films, such as evaporating deposition, sputtering deposition, ion plating, plasma enhanced chemical vapor deposition (PECVD), atmospheric pressure plasma deposition, sol-gel method and oxidation method etc. and the preparative techniques have obtained a certain research results so far. In this article, the principle and research status of different preparation technologies were summarized, and the application of silicon oxide films were discussed in aspects of packaging industry, microelectronics field, andoptical field. Besides, its application prospect is forecasted. Silicon oxide film swith more stable performance will gradually become industrialization and occupy the dominant position in surface modification for a long time.
\end{abstract}

Keywords: silicon oxide film; preparation methods; application; CVD; PVD.

\section{INTRODUCTION}

Silicon oxideis a widely used thin film material. It has many excellent properties such as anti-resistance, hardness, corrosion resistance, dielectric, optical transparency etc. It has been used in many fields $[1,17,18$, 20]. In microelectronic field, it is widely used as the most common dielectric. As silicon oxide has adjustable forbidden band width, it can be served as light absorption layer of the thin film of amorphous silicon solar cells to improve light absorption efficiency. It also can be used as the gate dielectric layer of MOS and CMOS devices or the thin film transistor. In optical field, it is utilized for passive or active optical devices, which not only have excellent light-admitting quality but also have other basic functions, such as electro-optic modulation and optical amplification. Therefore, silicon oxide can be used as waveguide film, AR coating and antireflection film[26]. In the packaging industry, silicon oxide film is used as a barrier layer of polymer packaging materials. Most of modern packaging materials cannot offer a sufficient barrier against permeation of gases, which will lead to a reduced self-life of food and drink. Just because of this, a silicon oxide film deposited on the surface of polymer packaging becomes popular and indispensable. Besides, silicon oxide film also can be used as a corrosion protective layer of metals. Because of the universal application of silicon oxide films in various fields, preparation of silica with high quality is always an important content of scientific research. Preparation methods of silica change with its different purposes and requirements $[3,7,9,15,20]$.At present, there are a lot of preparation methods of silica, mainly including physical vapor deposition (PVD), chemical vapor deposition (CVD), sol-gel method, oxidation method etc. Among them, physical vapor deposition (PVD) includes evaporating deposition, sputtering deposition and ion plating; chemical vapor deposition (CVD) mainly includes traditional chemical vapor deposition and plasma enhanced chemical vapor deposition (PECVD). In addition, atmospheric pressure plasma deposition technology is also a common coating technology, because it does not need to vacuum, suitable for mass deposition.

In this paper, the preparation methods and application research of silicon oxide films are reviewed, at the same time, its application prospect is discussed. 


\section{PREPARATION METHODS}

There are many methods for depositing silicon oxide film. Various preparation methods and different reaction systems vary with the different properties needed of silicon oxide. And each method has its advantages and disadvantages.

\section{A. $\quad$ Physical Vapor Deposition (PVD)}

Physical vapor deposition is one of the traditional vacuum deposition techniques. It includes vacuum evaporation, ion plating and sputtering deposition.

Vacuum evaporation is one of the earliest methods in physical vapor deposition technology. Its basic principle is to make metal, metal alloy or compounds evaporate under the vacuum condition and then deposit on the surface of substrate. According to different heating source, the vacuum evaporation can be divided into resistance wire evaporation and electron beam evaporation, as shown in Fig .1 and Fig .2.But the binding force between silica film and substrate is very weak by this depositing method. Besides, the composition of film is difficult to control due to the phenomenon of thermal decomposition.

In 1963,ion plating technology was proposed by Mattox of Sandia Company for the first time. Using some kind of plasma ionization technology make the atom of plating material ionize into ion partly under the vacuum condition, at the same time, produce many energetic neutral atom. And then a thin film deposit on substrate surface under the action of negative bias. This is the basic principle of ion plating. The deposited film has good adhesion performance by this method. But the device and the operation are complicated.

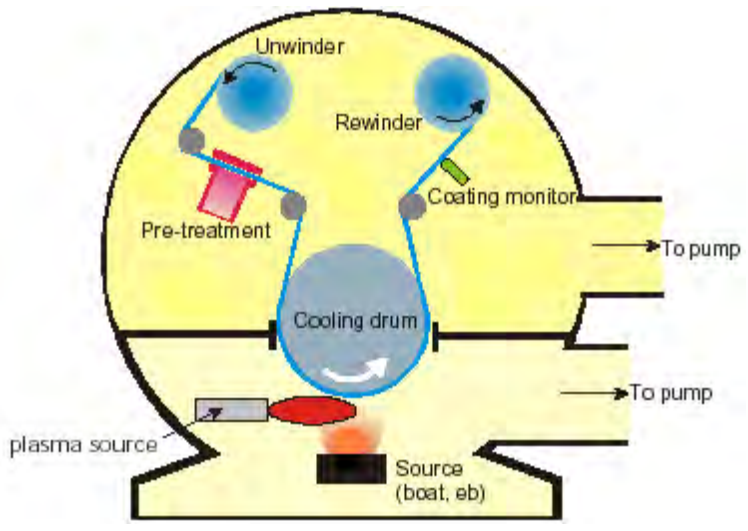

Figure 3.Resistance wire evaporation

Compared with other film preparation method, the film deposited by magnetron sputtering method has uniform thickness, and the controllability and repeatability are obviously improved. Feng Chen et al [10].deposited the porous Silicon oxide thin films by using radio-frequency magnetron sputtering method on the substrate of porous alumina membranes.

\section{B. Sol-gel Method}

Sol-gel is one of the most common methods of the preparation of inorganic membrane. Its raw material is metal alkoxide or inorganic salt which undergoes hydrolytic condensation reaction sand becomes sol under

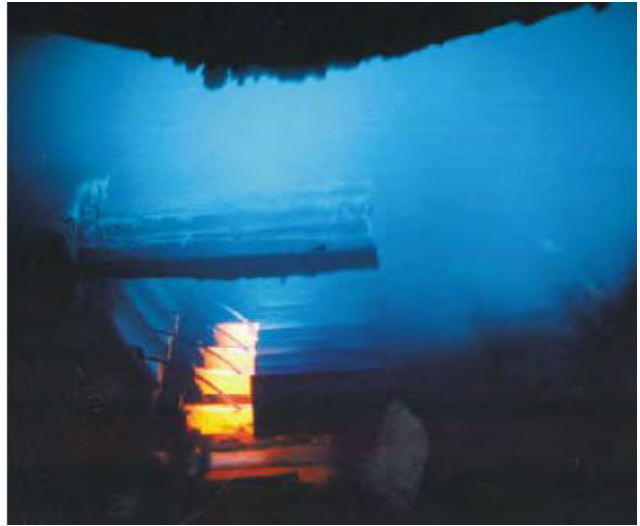

Figure 1. Electron beam evaporation

the certai

, dry,

roasting process etc. forms a porous inorganic membrane. Sol-gel is regarded as an effective method of preparation of porous ceramic membrane, because raw materials can be mixed at the molecular level in the method and the film can form at a low temperature.

\section{Thermal Oxidation Method}

The silicon can be oxidized into silicon oxide film under the high temperature about $900-1200^{\circ} \mathrm{C}$.Thermal oxidation method includes the wet oxygen oxidation, dry oxygen oxidation and steam oxidation. The time of forming Silicon oxide thin film about $10 \mathrm{~nm}$ under the dry oxygen atmosphere of high temperature is very short, and this time cannot be controlled for conventional resistance wire heating oxidation furnace. While the oxidation method with high temperature and low pressure can extend the oxidation time, but a long time high temperature process will cause redistribution. Therefore, rapid thermal oxidation (RTO) catches the attention. This process can take place at relatively high temperatures $\left(900-1100^{\circ} \mathrm{C}\right)$ for well controlled short periods of time. Y. L. Chiouet al.[12, 27]obtained the ultra-thin silicon oxide films with good performance by rapid thermal oxidation, and they also analyzed the growth characteristics of silicon oxide.

\section{Chemical Vapor Deposition (CVD)}

The gas phase reactants turn to solid undergoing chemical reaction and the solid deposits in heating surface of the solid matrix. Due to the CVD is a technology of preparing thin film through the chemical reactions, the film obtained has good characteristics in crystallization. Therefore, in large scale integrated circuit manufacturing technology, the CVD method has been used to deposit thin film[1-5].However CVD belongs to thermally

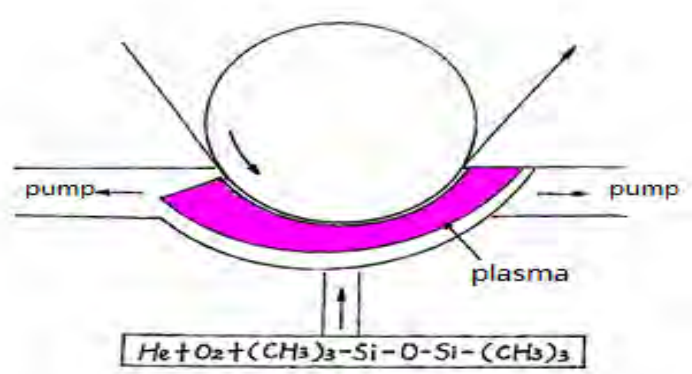

Figure 2. Plasma enhanced chemical vapor deposition 
activated methods, which require high temperatures in the process of coating. That makes CVD methods unacceptable for deposition on temperature-sensitive substrate materials[31].

Plasma enhanced chemical vapor deposition (PECVD) is a widely used technique for silica thin film deposition, as shown in Fig .3.Cold plasma processing generates reactive species that undergo a variety of reactions at very low temperature[23, 25].

The properties of PECVD-grown silica films are determined by the chemical compositions of reactants and other deposition conditions in the glow discharge. In the process of the preparation of silica films the choice of silicon sources is important. Commonly used silicon sources mainly have gaseous silane $(\mathrm{SiH} 4)$ or evaporated

liquids such as tetraethoxysilane (TEOS), tetramethylsilane (TMS), hexamethyldisiloxane (HMDSO), hexamethydisilazane (HMDSN) etc. [13, 35]. In recent decades, silane has been commonly used as silicon source when preparing silica thin films using the PECVD system[38]. But considering the safety and toxicity of silane, organo silicon precursors, such as tetramethylsilane (TMS) and tetraethoxysilane (TEOS) etc., which are easy to handle due to their chemical stability at room temperature have been adopted as alternatives for $\mathrm{SiH} 4[30]$. The coating composition is varied from $\mathrm{SiOxCyHz-like} \mathrm{to} \mathrm{inorganic} \mathrm{SiOx}$, which mainly influenced by the admixture of oxygen. The properties of inorganic $\mathrm{SiOx}$ films are observed to have higher permeation barrier properties. Y. Qi, Z. G. Xiao and T. D. Mantei[31] compared the deposition results fromhexamethyldisiloxane(HMDSO), octamethylcy-clotet rasiloxane(OMCTS), andtetramethylcyclotetra-siloxane(T MCTS). In their studies, growth rates and hardness increased with the $\mathrm{O} 2$ /precursor flow ratio at low to modest ratios. The highest growth rates were obtained with HMDSO, while the highest-quality coatings were grown from TMCTS, in terms of hardness and low carbon content. In addition, the way of deposition will also affect the mechanical properties of thin film, just as $\mathrm{V} \mathrm{Au}, \mathrm{C}$ Charles and R W Boswell[2] reported. In their works, they compared the stress of amorphous silicon oxide film in a single-layer continuous deposition with the multiple-layer intermittent or 'stop-start' depositions. They found that the stress of multiple-layer samples higher than the single-layer film at film thicknesses of less than $300 \mathrm{~nm}$, while there is little change in the film stress between the samples at $1 \mu \mathrm{m}$ film thickness. PECVD which can obtain the silicon oxide film with high density and good dielectric properties at low temperature is one of the main methods of depositing silicon oxide film. However, it must work at low pressure atmosphere and need corresponding vacuum system, which is expensive and complex. Besides, PECVD cannot deposit film in the complex surface. Therefore, it is a critical issue to find a suitable method, which can get low cost deposition of silica films with high quality and on a large scale. Fortunately, atmospheric pressure plasma deposition technology solves these problems perfectly.

Atmospheric plasma superficial treatments not only reduce costs but also increase the on-line processing capability, which endows $\mathrm{SiOx}$ barrier films with high flexibility and high mechanical resistance[24, 28, 29, 33, 34].That is important for packaging materials. Recently, atmospheric pressure discharges have drawn a lot of attention, and silica films have been successfully prepared by this method. D Trunec et al. [36] achieved the deposition of thin organo silicon films by burning nitrogen with a small admixture of hexamethyldisiloxane (HMDSO) using atmospheric pressure dielectric barrier discharge.Paolo Scopece et al.[33] successfully deposited $\mathrm{SiOx}$ thin films onto PP films by atmospheric pressure plasma jet (APPJ) in terms of gas permeation behavior. $\mathbf{J}$ Sch“afer et al.[32] also using APPJ $(27.17 \mathrm{MHz}$, Ar with 1\% HMDSO) studied for the deposition of thin silicon-organic film. These studies realized large-scale production to some extent, but the study by Xiaodong Zhu et al.[39] achieved this goal better. Xiaodong Zhu et al. have investigated a line-shaped atmospheric pressure filamentary plasma, which is developed to carry out the open air deposition of silica films from N2/hexamethyldisoxane (HMDSO) mixtures with or without adding oxygen. In their study, the important role of the oxygen in the open air reactor has been shown. And it reveals a potential of open air deposition Silicon oxide films at atmospheric pressure for large-scale deposition.

Besides, preventing oxidation silicon thin film from carbon pollution is also a problem needed to consider[8, $16,22]$. Theil et al. reported that the film obtained from PECVD with organosilicon precursor at room temperature was contaminated with carbon as high as $0.7 \%$. While though the study of Takaomi Matsutaniet al.[30], room-temperature preparation of silica films was achieved by low-energy ion-beam induced chemical vapor deposition (IBICVD) with a bubbling system for hexamethyldisiloxane (HMDSO).This kind of films have a smooth surface and a low carbon contamination of less than $0.1 \%$.

\section{APPLICATIONS}

\section{A. Application in the packaging industry}

With the development of the food and pharmaceutical industries during the last decades, packaging technology and application have seen several changes. In the food packaging sector, isotactic polypropylene (PP) and polyethylene terephthalate (PET) hold a prominent position because of their transparency, low specific weight and chemical inertness. But PP and PET cannot offer a sufficient barrier against permeation of gases through the polymer, which results poor protection of the packaged food[33].It has been proven that silicon oxide coating is useful in improving the gas barrier properties of plastic packaging. Besides, depositing a silicon oxide film at the surface of packaging materials is currently substituting Al-coated polymers because of the transparent and microwaveable properties of silica films[15]. Just as the excellent blocking performance, some developed countries such as Europe, Japan, the United States etc. have developed a series of silicon oxide composite film packaging to replace the Al-coated composite packaging materials and obtained the satisfactory results[21].The Coca-Cola Company (TCCC) working together with Krones $\mathrm{AG}$ had enabled the patenting of BESTPET ${ }^{\mathrm{TM}}$ (Barrier Enhanced Silica 
Treated PET), which is a revolutionary barrier technology for PET bottles. BESTPET ${ }^{\mathrm{TM}}$ made the shelf life of PET bottles increase more than doubles by reducing $\mathrm{CO} 2$ loss and oxygen pick-up.

\section{B. Application in microelectronic field}

Silicon oxide thin films have been widely used in microelectronics technology due to its superior electrical insulation and the feasibility of the technology. As the forbidden band of Silicon oxide is broad, Silicon oxide films can be used as the light absorption layer of amorphous silicon solar cell, in order to improve the light absorption collecting efficiency. Besides, it also can be used as the gate dielectric layer of CMOS devices, SiGe MOS devices and the thin film transistor (TFT) etc. in integrated circuit $[6,19]$. The silicon oxide films as a new type of dielectric material with low dielectric constant have many advantages, such as low loss and low power, high adhesion and high hardness, corrosion resistance and low water absorption, high stability and low shrinkage. At present, the dielectric constant of Silicon oxide dielectric layers is about 4.0. G. Borvon et al. [6]reported that the dielectric constant decreases with the concentration ratio of $\mathrm{Si}(\mathrm{CH} 3) 3 / \mathrm{Si}-\mathrm{O}-\mathrm{Si}$. In lithium ion battery, the diaphragm, which separates the positive and negative electrodes, prevents short circuit cause. Silicon oxide film deposited on the surface of the diaphragm (soft polyolefin) can improve the thermal stability of the surface wettability of diaphragm for the electrolyte. As a result, the performance of lithium battery is improved.

\section{Application in optical field}

With the rapid development of optical communication and integrated optics technology, optical waveguide of glass film is widely used in optical passive device and integrated optics. The preparation of optical waveguide thin film with good performance is crucial. Silica-based thin films are of low loss over a wide frequency range, ease of processing and thermal and mechanical stability, so they are utilized for passive or active optical devices in the field of optical communications[26].Furthermore, silicon oxide can also be used as waveguide film, AR coating and antireflection film in optical device.

\section{Application prospect}

In addition to the above mentioned fields, silicon oxide film also can be used as a corrosion protective layer of metals[11, 14, 37].All of these years, the research of protecting metals from corrosion have been the center of attention, because it prevents the use of some certain metals and alloys on strategic applications such as power system or transport[14]. At present, the most devices in power system are faced with the problem of the aging and corrosion in the surface of rubber protective layer, while applied the corrosion resistance property of silicon oxide, depositing a layer of silicon oxide in the surface of conductor is a kind of methods to solve this problem.

What is more, silicon oxide film can get low surface energy by controlling the growth of silicon oxide, making food packaging materials with super hydrophobic. This kind of packing materials can reduce the residues on food packaging, especially for viscous food such as yogurt, and avoid waste of foods.

\section{CONCLUSIONS}

The excellent performance of silicon oxide film has absorbed the wide attention in academia, and their preparative techniques have obtained a certain research results so far. It is expected that the preparation of silicon oxide thin film technology further mature and the silicon oxide film with more stable performance gradually become industrialization and broader application fields are developed.

\section{ACKNOWLEDGMENT}

The financial support of this work extended by the Science and Technology Project of State Grid Shanxi Electric Power Company (Grant 5205E01351DG) and the Science and Technology Project of State Grid Corporation (Grant FTZX201404) are gratefully acknowledged. Furthermore, we are grateful for the help of Plasma Laboratory in Beijing Institute of Graphic Communication.

\section{REFERENCES}

[1] I. Aharonovich, Y. Lifshitz, S. Tamir, "Growth mechanisms of amorphous $\mathrm{SiO}[\mathrm{sub} \mathrm{x}]$ nanowires". Applied Physics Letters 90, 263109 (2007).

[2] V. Au, C. Charles, R. W. Boswell, "Comparison of stress in single and multiple layer depositions of plasma-deposited amorphous silicon dioxide". Journal of Physics D: Applied Physics 39, 164-171 (2006).

[3] L. Bárdos, H. Baránková, "Cold atmospheric plasma: Sources, processes, and applications". Thin Solid Films 518, 6705-6713 (2010).

[4] T. Belmonte, G. Henrion, T. Gries, "Nonequilibrium Atmospheric Plasma Deposition". Journal of Thermal Spray Technology 20, 744-759 (2011)

[5] I. Blaszczyk-Lezak et al., "Remote nitrogen microwave plasma chemical vapor deposition from a tetramethyldisilazane precursor. 1 . Growth mechanism, structure, and surface morphology of silicon carbonitride films". Thin Solid Films 497, 24-34 (2006).

[6] G. Borvon, A. Goullet, X. Mellhaoui, N. Charrouf, A. Granier "Electrical properties of low-dielectric-constant films prepared by PECVD in O2/CH4/HMDSO". Materials Science in Semiconductor Processing 5, 279-284 (2002).

[7] Z. Cao, X. Zhang, "Size-dependent creep behaviour of plasma-enhanced chemical vapour deposited silicon oxide films". Journal of Physics D: Applied Physics 39, 5054-5063 (2006).

[8] R. P. Cardoso, T. Belmonte, F. Kosior, G. Henrion, E. Tixhon, "High-rate deposition by microwave RPECVD at atmospheric pressure". Thin Solid Films 519, 4177-4185 (2011).

[9] "Characterization and tribological investigation of $\mathrm{SiO} 2$ and $\mathrm{La} 2 \mathrm{O} 3$ sol-gel films".

[10] F. Chen, A. H. Kitai, "Growth of nanoporous silicon dioxide thin films using porous alumina substrates". Thin Solid Films 517, 622-625 (2008).

[11] Y. Cheng, X. Pang, K. Gao, H. Yang, A. A. Volinsky, "Corrosion resistance and friction of sintered $\mathrm{NdFeB}$ coated with $\mathrm{Ti} / \mathrm{TiN}$ multilayers". Thin Solid Films 550, 428-434 (2014).

[12] Y. L. Chiou, C. H. Sow, G. Li, K. A. Ports, "Growth characteristics of silicon dioxide produced by rapid thermal oxidation processes". Applied Physics Letters 57, 881 (1990).

[13] M. Deilmann, M. Grabowski, S. Theiß, N. Bibinov, P. Awakowicz, "Permeation mechanisms of pulsed microwave plasma deposited silicon oxide films for food packaging applications". Journal of Physics D: Applied Physics 41, 135207 (2008). 
[14] A. Delimi, Y. Coffinier, B. Talhi, R. Boukherroub, S. Szunerits, "Investigation of the corrosion protection of SiOx-like oxide films deposited by plasma-enhanced chemical vapor deposition onto carbon steel". Electrochim Acta 55, 8921-8927 (Dec 1, 2010).

[15] "Diagnostics and insights on PECVD for gas-barrier coatings*".

[16] C. Gerhard et al., "Atmospheric Pressure Plasma Treatment of Fused Silica, Related Surface and Near-Surface Effects and Applications". Plasma Chemistry and Plasma Processing 33, 895-905 (2013).

[17] D. Gitlin, J. Karp, B. Moyzhes, "Graded tunnelling barrier and oxygen concentration in thermally grown ultrathin $\mathrm{SiOx}$ gate oxide". Journal of Physics D: Applied Physics 40, 2143-2149 (2007).

[18] C. Gravalidis, M. Gioti, A. Laskarakis, S. Logothetidis, "Real-time monitoring of silicon oxide deposition processes". Surface and Coatings Technology 180-181, 655-658 (2004).

[19] A. Grill, D. A. Neumayer, "Structure of low dielectric constant to extreme low dielectric constant $\mathrm{SiCOH}$ films: Fourier transform infrared spectroscopy characterization". Journal of Applied Physics 94, 6697 (2003).

[20] B. Hoex et al., "High-rate plasma-deposited $\mathrm{SiO}[$ sub 2] films for surface passivation of crystalline silicon". Journal of Vacuum Science \& Technology A: Vacuum, Surfaces, and Films 24, 1823 (2006)

[21] D. G. Howells et al., "Mechanical properties of SiOx gas barrier coatings on polyester films". Surface and Coatings Technology 202, 3529-3537 (2008).

[22] N. Jidenko, C. Jimenez, F. Massines, J. P. Borra, "Nano-particle size-dependent charging and electro-deposition in dielectric barrier discharges at atmospheric pressure for thin SiOx film deposition". Journal of Physics D: Applied Physics 40, 4155-4163 (2007).

[23] J.-K. Kim, S.-H. Jeong, B.-S. Kim, S.-H. Shim, "Characterization and preparation of $\mathrm{SiO} 2$ and $\mathrm{SiOF}$ films using an RF PECVD technique from TEOS/O2 and TEOS/O2/CF4 precursors". Journal of Physics D: Applied Physics 37, 2425-2431 (2004).

[24] Y. Kusano, "Atmospheric Pressure Plasma Processing for Polymer Adhesion: A Review". The Journal of Adhesion 90, 755-777 (2014).

[25] C. Lasorsa, P. J. Morando, A. Rodrigo, "Effects of the plasma oxygen concentration on the formation of $\mathrm{SiOxCy}$ films by low temperature PECVD". Surface and Coatings Technology 194, 42-47 (2005)

[26] W. T. Li, R. Boswell, M. Samoc, A. Samoc, R. P. Wang, "The effect of defects on the optical nonlinearity of thermally poled SiOx thin films". Thin Solid Films 516, 5474-5477 (2008).
[27] C. Lin, Y. Wang, Y. Yen. (United Microelectronics Corp (Umec-C)).

[28] X. Lu, "Review on atmospheric pressure pulsed DC discharge". Scientia Sinica Physica, Mechanica \& Astronomica 41, 801 (2011).

[29] S. Martin, "Atmospheric pressure PE-CVD of silicon based coatings using a glow dielectric barrier discharge". Surface and Coatings Technology 177-178, 693-698 (2004).

[30] T. Matsutani, T. Asanuma, C. Liu, M. Kiuchi, T. Takeuchi, "Deposition of $\mathrm{SiO} 2$ films by low-energy ion-beam induced chemical vapor deposition using hexamethyldisiloxane". Surface and Coatings Technology 177-178, 365-368 (2004).

[31] Y. Qi, Z. G. Xiao, T. D. Mantei, "Comparison of silicon dioxide layers grown from three polymethylsiloxane precursors in a high-density oxygen plasma". Journal of Vacuum Science \& Technology A: Vacuum, Surfaces, and Films 21, 1064 (2003).

[32] J. Schäfer, R. Foest, A. Quade, A. Ohl, K. D. Weltmann, "Local deposition of SiOxplasma polymer films by a miniaturized atmospheric pressure plasma jet (APPJ)". Journal of Physics D: Applied Physics 41, 194010 (2008).

[33] P. Scopece et al., "SiOx-Based Gas Barrier Coatings for Polymer Substrates by Atmospheric Pressure Plasma Jet Deposition". Plasma Processes and Polymers 6, S705-S710 (2009)

[34] S. A. Starostin et al., "On the formation mechanisms of the diffuse atmospheric pressure dielectric barrier discharge in CVD processes of thin silica-like films". Plasma Sources Science and Technology 18,045021 (2009)

[35] D. Theirich, C. Soll, F. Leu, J. Engemann, "Intermediate gas phase precursors during plasma CVD of HMDSO". Vacuum 71, 349-359 (2003).

[36] D. Trunec et al., "Deposition of hard thin films from HMDSO in atmospheric pressure dielectric barrier discharge". Journal of Physics D: Applied Physics 43, 225403 (2010).

[37] C. Vautrin-Ul, F. o. Roux, C. Boisse-Laporte, J. L. Pastol, A. Chausse, "Hexamethyldisiloxane (HMDSO)-plasma-polymerised coatings as primer for iron corrosion protection: influence of RF bias". Journal of Materials Chemistry 12, 2318-2324 (2002).

[38] C.-Y. Wu, W.-C. Chen, D.-S. Liu, "Surface modification layer deposition on flexible substrates by plasma-enhanced chemical vapour deposition using tetramethylsilane-oxygen gas mixture". Journal of Physics D: Applied Physics 41, 225305 (2008).

[39] X. Zhu, F. Arefi-Khonsari, C. Petit-Etienne, M. Tatoulian, "Open Air Deposition of $\mathrm{SiO}_{2}$ Films by an Atmospheric Pressure Line-Shaped Plasma". Plasma Processes and Polymers 2, 407-413 (2005). 